Journal of Mathematics and Statistics 3 (1): 12-14, 2007

ISSN 1549-3644

(C) 2007 Science Publications

\title{
Flow of Temperature-dependent Viscous Fluid Between Parallel Heated Walls: Exact Analytical Solutions in the Presence of Viscous Dissipation
}

\author{
K. S. Adegbie and F.I. Alao \\ Department of Mathematical Sciences, Federal University of Technology \\ P.M.B.704, Ondo state, Akure, Nigeria
}

\begin{abstract}
We examine steady incompressible flow of viscous liquids between parallel heated walls of plane Couette device. The temperature of the upper and lower walls of the device are maintained at $\mathrm{T}=$ $T_{b}$ and $T=T_{0}$ respectively. Of a particular interest are exact analytical solutions of the coupled nonlinear differential equations resulting from plane Couette flow obtained for the temperature and velocity distributions respectively. The criterion for which the solutions are valid was determined by the temperature difference, $\alpha$, between the upper and lower walls. The analysis reveals that the shear stress obtained at the walls exists when the temperature difference $\alpha>0$.
\end{abstract}

Key words: Steady incompressible flow, Newtonian viscous liquids, coupled nonlinear differential equations, exact analytical solutions, Reynold's model viscosity, viscous dissipation

\section{INTRODUCTION}

The study of flow of viscous fluid with temperature dependent properties is of great importance in lubrication and tribology, food processing, instrumentation and viscometry. However, viscous heating is always a possible and frequently significant, source of error in viscometric measurement at high shear rates in instrumentation and viscometry. Bird et $a l .{ }^{[1]}$, Turian and $\operatorname{Bird}^{[2]}$ and Turian ${ }^{[3]}$ have presented a methodology for obtaining approximate analytical solutions to the problem of combine flow and heat transfer in planar Couette flow when both the viscosity and thermal conductivity are polynomial functions of temperature. Papathanasiou ${ }^{[4]}$ developed second-order series solution for flow in circular Couette with walls maintained at constant temperature, for material whose viscosity and thermal conductivity can be expressed as polynomial functions of temperature with arbitrary coefficients. Davis et al. ${ }^{[5]}$ studied steady parallel flows of Newtonian liquids that have temperature dependent viscosities and substantial viscous heat generation. They presented shear stress versus shear rate characteristics and found that activation energy parameter affects the results considerably. They observed that shear-stress rate graphs are either monotonic or there exists large jump in shear rate and heat transfer at the walls. Adler ${ }^{[6]}$ investigated the thermal stability of a reactive viscous flow. He considered the steady developed flow between symmetrically parallel heated walls and used a power series in a defined viscous heating parameter to obtain an expression for critical Frank-Kamenetskii parameter in series form. Johns and Narayanan ${ }^{[7]}$ considered frictional heating in plane Couette flow. There result was this: if the wall speed is the control variable there are no points of neutral stability; if the wall speed is the control variable the nose of the curve is a point of neutral stability. This supports their conviction that in a physical experiment the wall speed must be the control variable, it cannot be the wall stress. Because the wall stress plays the same role here as does the FrankKamenetskii number in thermal ignition they concluded that thermal ignition is not a good model of fluid frictional heating. If the viscosity of a fluid in plane Couette flow decreases exponentially as its temperature increases, the curve of wall speed versus wall stress has two branches, separated by a turning point. Subrahmaniam et al ${ }^{[8]}$ demonstrated that the upper branch of this curve is stable to zero wave number disturbances in case the power supplied to move the wall is the input variable and held fixed. Yürüsoy and Pakdemiril ${ }^{[9]}$ considered the flow of a third-grade fluid in a pipe with heat transfer. Constant viscosity, Reynold's model viscosity and Vogel's model viscosity cases are treated separately. Approximate analytical solutions are presented for each case using perturbations. The criteria for which the solutions are valid are determined for the dimensionless parameters involved. The analytical solutions are contrasted with the finite difference solutions given in Massoudi and Christie $^{[10]}$. Recently, Adegbie and Alao ${ }^{[11]}$ studies steady-state flow of Newtonian viscous liquid with exponential temperature-dependent viscosity and substantial viscous heat generation in a symmetrically heated channel. The coupled non-linear momentum and energy equations arising in planar Poiseuille flow are solved numerically using finite difference scheme techniques. The effects of flow controlling parameters, such as, viscous heating parameter, temperature

Corresponding Author: $\quad$ K. S. Adegbie, Department of Mathematical Sciences, Federal University of Technology, P.M.B.704, Ondo state, Akure, Nigeria, Tel: +234-805-665-5749 
difference and pressure-gradient parameter on the velocity and temperature profiles are analysed. The present contribution studies steady-state flow of Newtonian liquid with exponential temperaturedependent viscosity and substantial viscous heat generation between symmetrically parallel heated walls with walls at different temperatures. Exact analytical solutions for temperature and velocity profiles in the plane Couette device are obtained. The analysis reveals that the criterion for which the solutions exist depends on the temperature difference between the upper and lower walls of the Couette device, that is, $\alpha>0$. The shear stress obtained at the wall also depends on the temperature difference, $\alpha$.

Mathematical formulation: We consider steady flow of Newtonian viscous liquids characterized by temperature dependent viscosity and viscous dissipation due to a high velocity gradient of the flow between the walls. The main physical assumptions of the suggested problem are the following. The flow is incompressible and fully developed. The body forces and pressure changes influence are negligible. We presuppose there is no-slip at the boundary. The dependence of thermal conductivity on temperature is neglected. The upper wall temperature is higher than the lower wall temperature, which is assumed to be the initial temperature of the fluid. The viscosity depends on temperature in an exponential manner, Reynold's Model Viscosity,

$\bar{\mu}=\mu_{0} \exp (-T)$

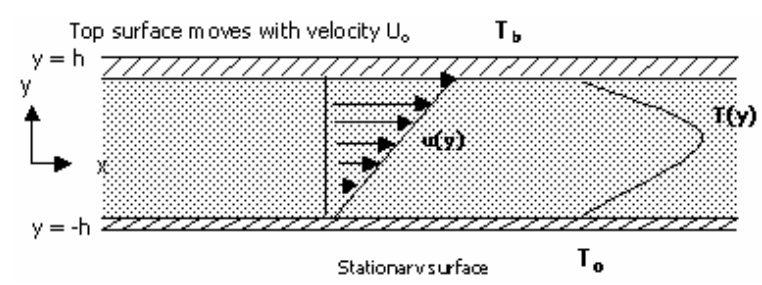

Fig. 2.1: Schematic description of physical model

Under these assumptions, the system of governing dimensionless momentum balance and energy balance equations takes the following form

$$
\begin{aligned}
& \frac{d}{d r}\left[\exp (-\alpha \theta) \frac{d u}{d r}\right]=0 \\
& \frac{d^{2} \theta}{d r^{2}}+B_{r m} \exp (-\alpha \theta)\left(\frac{d u}{d r}\right)^{2}=0
\end{aligned}
$$

subject to the following boundary conditions:

$$
\left.\begin{array}{l}
u(-1)=0, u(1)=1 \\
\theta(-1)=0, \theta(1)=0
\end{array}\right\} .
$$

The above coupled non-linear flow governing equations were made dimensionless using
$\theta=\frac{T-T_{0}}{T_{b}-T_{0}}, u=\frac{\bar{u}}{U_{0}}, r=\frac{y}{h}, \mu=\frac{\bar{\mu}}{\mu_{0}}$

The dimensionless parameters involve in equations $(2.2)-(2.3)$ are

$$
\alpha=T_{b}-T_{0}, B_{r m}=\left(\frac{\mu_{0} U_{0}^{2}}{\alpha \lambda}\right) \exp \left(-T_{0}\right)
$$

where $B_{r m}$ is the modified Brinkman number, $B_{r}=\left(\mu_{0} U_{0}^{2} / \alpha \lambda\right)$ is Brinkman number, which is a measure of heat generated by viscous heating as compare to the heat conducted from the impressed temperature difference $\alpha$ between the upper and lower walls respectively through the viscous liquid. $\lambda$ is the thermal conductivity, $\theta$ is the dimensionless temperature, $u$ is the velocity parallel to the planes, $U_{0}$ is the constant velocity at moving surface and $\mu_{0}$ is the viscosity at $T_{0}$.

Method of solution: Here, we consider the solutions of flow of viscous liquid with a high velocity gradient between the walls of plane Couette device at different temperature when its viscosity is an exponential function of temperature.

Integrating (2.2) with respect to $r$ yields

$\frac{d u}{d r}=a \exp (\alpha \theta)$

where a is constant of integration.

Substituting (3.1) into (2.3), we obtain

$\frac{d^{2} \theta}{d r^{2}}+\sigma \exp (\alpha \theta)=0$

Where $\sigma=a^{2} B_{r m}$.

From symmetry consideration, we need only solve the equation (3.2) with the combined boundary conditions:

$\theta=0$ at $r=1, \frac{d \theta}{d r}=0$ at $r=0$.

In order to find the criterion, which satisfies the boundary conditions, we use the temperature at the middle of the device $\theta_{m}$ (at this point $\theta_{m}$ is unknown) as a parameter and solve the (3.2) subject to the conditions:

$\theta=\theta_{m}$ and $\frac{d \theta}{d r}=0$ at $r=0$.

Multiplying both sides of (3.2) by $2(d \theta / d r)$ and integrating yields

$\left(\frac{d \theta}{d r}\right)^{2}=2 \delta\left[\exp \left(\alpha \theta_{m}\right)-\exp (\alpha \theta)\right]$ 
where $\delta=a^{2} \mu_{0} U_{0}^{2} \exp \left(-T_{0}\right) / \lambda \alpha^{2}$.

Taking the square root of (3.5) and integrating again, we get

$\exp [\alpha \theta(r)]=\exp \left(\alpha \theta_{m}\right) / \cosh ^{2}(b \pm \beta r)$

where $\beta=\alpha \sqrt{\frac{\delta \exp \left(\alpha \theta_{m}\right)}{2}}$ and $b$ is constant of integration.

Employing the condition of symmetry $d \theta / d r=0$ at $r=0$ in (3.3), constant $b$ must be equal to zero and thus we have

$\exp [\alpha \theta(r)]=\exp \left(\alpha \theta_{m}\right) / \cosh ^{2}(\beta r)$

Applying the boundary condition $\theta=0$ at $r=1$ in (3.7) gives

$\theta_{m}=2 \ln \cosh (\beta) / \alpha$

Substituting (3.8) into (3.7) and taking the natural logarithm of both sides, we obtain

$\theta=\theta_{m}+(1 / \alpha) \ln \sec h^{2}\left(\alpha \sqrt{\frac{\delta \exp \left(\alpha \theta_{m}\right)}{2}} r\right)$

Applying the boundary conditions $\theta( \pm 1)=0$, we have

$\delta=2 \exp \left(-\alpha \theta_{m}\right)\left\{(1 / \alpha) \cosh ^{-1}\left[\exp \left(\alpha \theta_{m} / 2\right)\right]\right\}^{2}$

Now, substitution of $\theta$ from (3.9) into (3.1) follows by integration gives

$u=\frac{1}{2}\left\{\operatorname{coth}\left(\alpha \sqrt{\frac{\delta \exp \left(\alpha \theta_{m}\right)}{2}}\right) \tanh \left(\alpha \sqrt{\frac{\delta \exp \left(\alpha \theta_{m}\right)}{2}} r\right)+1\right\}$,

upon using the boundary conditions $u(-1)=0$ and $u(1)=1$ (which follow from (2.3)).

For the purpose of analysis, the criterion for which the solutions in (3.9), (3.10) and (3.11) exist depends on the temperature difference between the outer and inner surfaces of the channel, that is, $\alpha>0$. The dimensionless shear stress $\tau_{w}$ at the wall is obtained as

$\tau_{w}=\alpha \sqrt{\frac{\delta \exp \left(\alpha \theta_{m}\right)}{2}} \exp \left(-T_{0}\right) \operatorname{coth}\left(\alpha \sqrt{\frac{\delta \exp \left(\alpha \theta_{m}\right)}{2}}\right)$

This shows that $\tau_{w}$ vanishes when $\alpha=0$. But this can never occur since the validity of the model is based on the tacit assumption that $\alpha>0$.

\section{CONCLUSION}

Steady flow of Newtonian fluid with exponential temperature dependent viscosity and viscous dissipation in symmetrically parallel-heated walls is investigated. The exact analytical solutions for temperature and velocity profiles in the plane Couette device are obtained. The analysis reveals that the criterion for which the solutions exist depends on the temperature difference between the upper and lower walls of the Couette device, that is, $\alpha>0$. The shear stress obtained at the wall also depends on the temperature difference, $\alpha$. It is found that the behaviour of the solutions is replica of some of the problems in lubrication, viscometry and engineering flows such as pumping of oil in pipes, flow of liquids in open channels and extrusion of plastics through dies.

\section{REFERENCES}

1. Bird, R.B., W.E. Stewart and E.N. Lightfoot, 1960. Transport Phenomena. John Wiley and Sons, New York.

2. Turian, R.M. and R.B. Bird, 1963. Viscous heating in the cone-and-plate viscometer-II. Newtonian fluids with temperature-dependent viscosity and thermal conductivity. Chem. Engg. Sci., 18: 689696.

3. Turian, R.M., 1965. Viscous heating in the coneand-plate viscometer-III. Non-Newtonian fluids with temperature-dependent viscosity and thermal conductivity. Chem. Engg. Sci., 20: 771-781.

4. Papathanasiou, T.D., 1997. Circular Couette flow of temperature-dependent materials: Asymptotic solutions in the presence of viscous heating. Chem. Engg. Sci., 52: 687-699.

5. Davis, S.H., G.A. Kriegsmann, R.L. Laurence and S. Rosenblat, 1983. Multiple solutions and hysteresis in steady parallel viscous flows. Physics of Fluid, 26: 1177-1182.

6. Adler, J., 1975. Thermal explosion theory for reactive flow between parallel heated wall. Combustion and Flame, 24: 151-158.

7. Johns, L.E. and R. Narayanan, 1997. Frictional heating in plane Couette flow. Proc. Royal Soc. London A, 453: 1653-1670.

8. Subrahmaniam, N., L.E. Johns and R. Narayanan, 2002. Stability of frictional heating in plane Couette flow at fixed power input. Proc. Royal Soc. London A, 458: 2561-2569.

9. Yürüsoy, M. and M. Pakdemirli, 2002. Approximate analytical solutions for the flow of a third-grade fluid in a pipe. Intl. J. Non-linear Mech., 37: 187-195.

10. Massoudi, M. and I. Christie, 1995. Effect of variable viscosity and viscous dissipation on the flow of a third-grade fluid in a pipe. Intl. J. NonLinear Mech., 30: 687-699.

11. Adegbie, K.S. and F.I. Alao, 2006. Flow of a Newtonian fluid in a symmetrically heated channel: Effect of viscosity and viscous dissipation. Mathematical Problems in Engineering, 2006: 1-7. 